\title{
Integration Of Information Technology And Simulation For Managing Manufacturing-Logistics Network
}

Brian D. Fitzpatrick, Rockhurst University, USA Shahid I. Ali, Rockhurst University, USA

\begin{abstract}
This paper helps investigate the integration of information technology (IT) and simulation in order to help understand how to set reliable delivery dates in manufacturing-logistics networks. This integration is necessary to assist organizations involved in the network to help plan and control their operations more efficiently. The authors reviewed previous work in the areas of IT-oriented logistics, real time simulation, and due date assignment. We describe a general architecture for a real-time simulation-based system and create a prototype based on our architecture. An application of the prototype in a small manufacturing company is employed. The prototype implemented was for a local manufacturer of made-to-order safety windows with three manufacturing plants and a one office location, all within a 50-mile radius. The prototype for this company was developed utilizing a Boreland's Delphi 3 application development tool. The reason that a distributed simulation model was not implemented was that we focused on validating the most critical components of our architecture using a simple prototype as our initial validation effort. The following possible advantages of our architecture were identified:

* The architecture can use the most up-to-date operational data to make decisions regarding delivery date assignment and network management.

* The architecture can support both central and distributed environments.

* The prototype developed based on the architecture could assign tight delivery dates.

* The prototype could be used to maintain or increase the level of on-time deliveries by monitoring the operations.

* The prototype could generate options on delivery dates and cost based on routings/priority and transportation service options.
\end{abstract}

Keywords: Just-In-Time (JIT) Systems, information technology (IT), Electronic Data Interchange (EDI), real-time simulation, due date assignment

\section{INTRODUCTION}

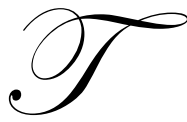

he globalization of information technology (IT) in manufacturing organizations allows a manufacturer to make their products at multiple locations. Parts of products may now be manufactured at locations anywhere in the world and transported several times until they are assembled as the final products. Then, the final products are delivered to customers who may be scattered around the world. Under this circumstance, it is critical to set reliable delivery dates of parts and final products for this network of transportation systems in order to efficiently manage operations and satisfy customer requirements simultaneously. This is especially important in organizations that implement Just-In-Time (JIT) systems.

IT has been extensively used to improve logistics or supply chain management systems. Networking suppliers, manufacturing facilities and customers using IT is not a novel idea to supply chain management. General Motors' Saturn operation uses a central computer that is networked with its suppliers in order to direct trucks to 
deliver parts at precise times to the docks of over 56 receiving factories (Henkoff, 1994). The achievement of this kind of JIT delivery requires tight integration of logistics with an efficient manufacturing plan and control. Each manufacturing facility involved in the logistics network should have the ability to set reliable delivery dates and control its operations to make the delivery on time. Currently, not many studies focus on how to use IT and simulation to manage logistics and manufacturing facility operations at the same time.

This paper presents a part of a study that investigates the integration of IT and simulation to set reliable delivery dates in manufacturing-logistics networks. This integration makes it possible for organizations involved in the network to plan and control their operations more efficiently. Real-time simulation used in an IT-networked supply chain provides a method to establish reliable delivery due dates. Also, this approach allows to generate several delivery date options with different cost structures, considering different production priorities and different modes of transportation.

This paper first briefly discusses previous work in the areas of IT-oriented logistics, real time simulation, and due date assignment. Next, we describe a general architecture for the real-time simulation-based system that support real-time delivery date decision-making and relating facility operations management in manufacturinglogistics network. Third, we describe a prototype that has been developed based on our architecture. The fourth section presents the application of the prototype in a small manufacturing company. The final section of the paper discusses a summary and suggests future research areas.

\section{BACKGROUND}

The use of networking and telecommunication technologies, such as Electronic Data Interchange (EDI), in logistics has been recognized as a competitive advantage by many organizations (Henkoff, 1994). Through IT, suppliers, manufacturers, and customers are integrated into a logistics-manufacturing network for efficient supply chain management. More recent research identified that the global nature of logistics now requires information systems that enhance inventory control, track orders and materials and monitor resource utilization (Lewis and Talalayesvsky, 1997). Also, surveys show that the trend to implement information systems, like EDI, will continue in the area of motor carriers (Murphy and Daley, 1996). In sum, IT has gained great importance in the control and management of manufacturing-logistics networks.

At the same time, simulation has been used for logistics planning for more than 20 years (Ballou, 1992). Integration of IT and simulation has also been recognized as an important role for real-time control (Harmonosky, 1990; McConnell and Medeiros, 1992; Rohrer, 1997). The integration of IT, with simulation for decision-making, is called real-time simulation. To apply real-time simulation in logistics networks, the implementation of sophisticated networked information systems is essential. This is because real-time simulation is based on the ability to obtain the real-time data needed to update the simulation model. Simulation-based real-time control for logistics network uses a simulation model initialized to the system's current status and a number of 'what-if' scenarios can be evaluated to make decisions (McConnell and Medeiros, 1992).

The decisions on which this research focuses are delivery date assignment and facility operations management to make the delivery on time. Delivery date assignment itself is a difficult problem given the dynamic nature of most manufacturing environments. In many Material Requirement planning environments, due dates are determined based on the estimated flow time of an order, plus some slack (Fry, Philipoom, and Markland, 1989). Several due date assignment methods have been proposed (Chang, 1996).

This paper integrates the concepts discussed above to propose a general architecture for a real-time simulation-based manufacturing-logistics management system. A system developed based on the architecture should be able to help manufacturers in logistics networks to make decisions regarding delivery date forecasting and facility operations management. In this architecture, the "up-to-date" information from the manufacturing centers, the transportation services, and the raw material suppliers (the manufacturing-logistics network) is utilized in order to forecast delivery dates more accurately and mange the facility operations to meet the due dates. 


\section{A GENERAL ARCHITECTURE FOR A REAL-TIME SIMULATION-BASED MANUFACTURING-LOGISTICS MANAGEMENT SYSTEM}

Delivery date assignment and relating facility operations management in logistics-manufacturing networks is a complex problem. First, this study identified the desired functionality of a system that handles delivery date assignments and facility operations control. Figure 1 shows relationships among manufacturers, raw-material suppliers, transportation services and customers located in multiple sites involved in the logistics network. As described in Hameri and Paatela (1995), each of these units can be connected in the multi-modal manner. This means that transportation service that connects two points may be a combination of road, railway, maritime, or air carriers. Each of the combinations has different costs, schedules/availability, lead times, and reliability characteristics. Similarly, each manufacturing facility has its associated characteristics, such as raw material on hand, lead times variability, and current jobs in process/schedules. Also, customers can be scattered all over a country or the world.

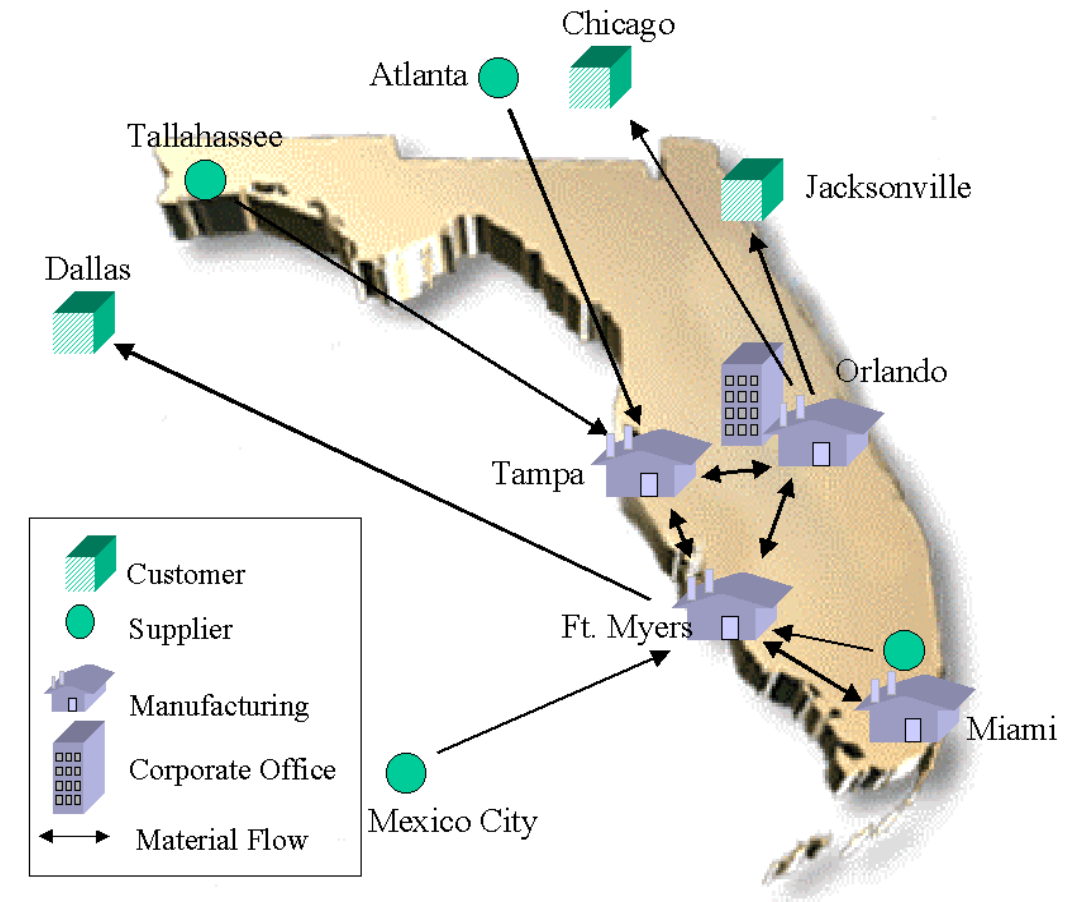

Figure 1: Material Flows among Organizational Units in Manufacturing-Logistics Network.

\subsection{Desired Functionality of Delivery Date Assignment and Facility Operation Management}

Under the circumstance described above, the process of simulation is initiated by changes in status of the logistics network. Examples of the changes in the network include arrival of new customer orders or changes in manufacturing or transportation resources. In the case that a new order changes the status of the network, the information about the new order is entered into the system. Then, the system obtains the current status of the network if it has not already been in the system. The system executes simulations of the new order under several scenarios to generate cost/delivery date options. The cost/delivery date options are offered to the customer and the customer selects the best option for him/her. The customer's selection is forwarded as routing/scheduling/material flow instructions to the organizational units involved in the network

In the case that changes are caused by unforeseen changes in the network (i.e. a significant delay in an assembly line or transportation service delay caused by accidents in a route), the system must be able to receive those changes as they occur. Then the system runs simulations to find the effect of the changes using the updated 
status of the network. If the simulation results show that some of the currently processed orders are delayed, corrective actions must be taken to meet the promised delivery date. To speed up the process, additional resources may be allocated, a higher priority in manufacturing queues may be used, or quicker transportation modes must be used. Thus, the system should be able to accept all necessary changes in the network operations as inputs and run simulations to find out the best possible corrective actions given the delivery date. The corrective actions selected must be disseminated to the organizational units affected by the changes. If changes are not accepted by some of the organizational units, different options must be offered. When the changes are accepted by all of the organizational units, the changes are forwarded as formal instructions. If late delivery is not avoidable, the earliest possible delivery date must be produced through the simulations. Then, this new delivery date must be communicated to the customers. This undesired situation must be identified as early as possible. Therefore, the system must continuously update the status of the network and simulate the effect of changes on the exiting orders. This functionality helps achieve efficient resource allocation and effective management of manufacturing facilities.

\subsection{Architecture of Real-Time Simulation-Based Manufacturing-Logistics Management System}

For the system to perform the desired functionality discussed in the previous section, it must have a set of components that work together. The components must be arranged in a certain way to achieve the overall goal of the system; in this case, assignment of delivery date and estimation of the effect of changes occurred in the logistics network on delivery date. Figure 2 presents the components of the system and their relationships. This architecture has been expanded from the one proposed by Roger and Gordon (1993) because their architecture includes only factory floor information. This is not sufficient to manage the entire manufacturing-logistics network that is tightly integrated. The extension has been done to include all the supply chain elements that critically affect the operations of the network.

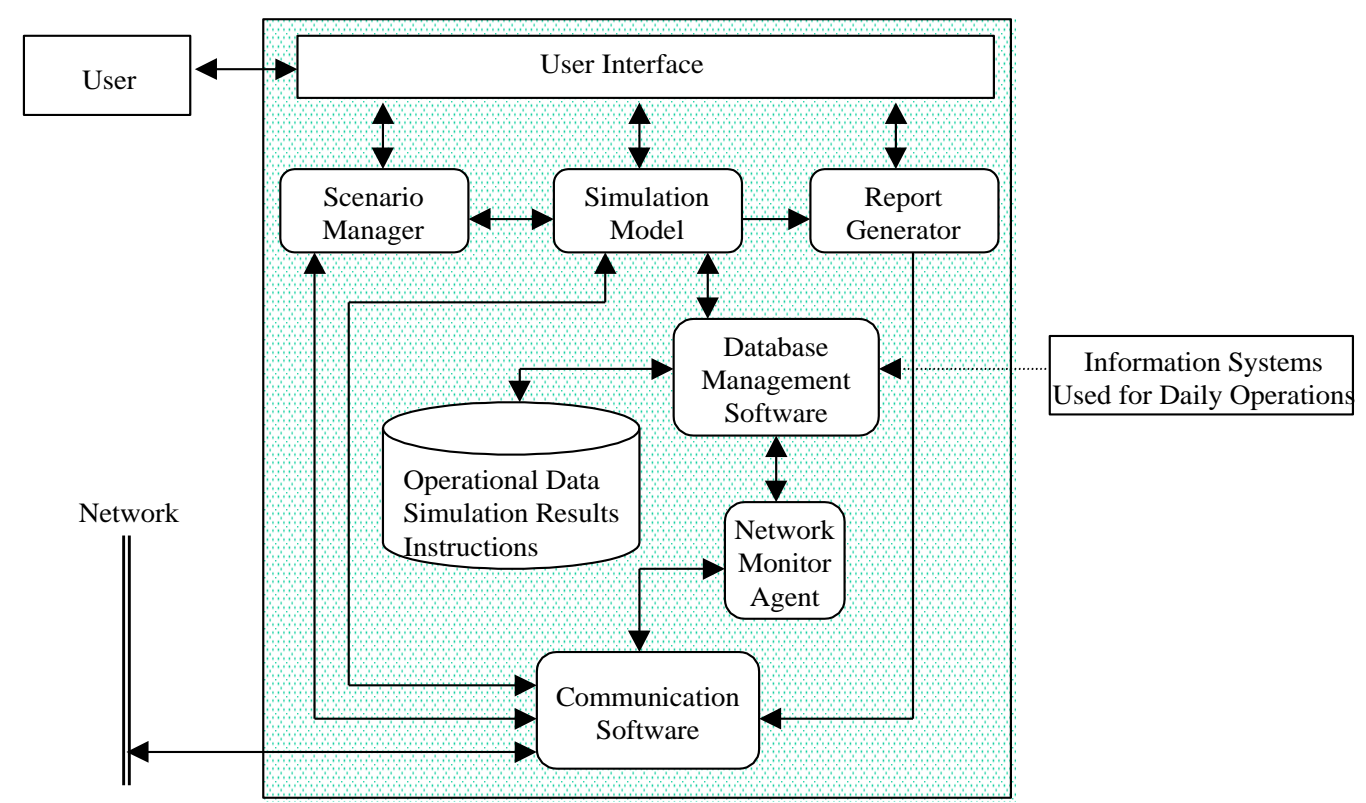

Figure 2: Architecture of Real-Time Simulation-Based Manufacturing-Logistics Management System

The major components of the system are:

- Scenario Manager maintains the pre-defined scenarios used in simulation to produce cost/delivery date options. The logistics analyst can specify or modify scenarios through the user interface. He/she also can define and modify scenarios stored in remote sites through communications software. The number of scenarios should be predetermined because there can be quite a large number of scenarios in a complicated logistics network. Each scenario requires additional run time. Also, the logistics analyst, from his/her 
experience, usually has extensive knowledge that some scenarios do not work well in practice. The pre-set scenarios will consist of variations in the transportation methods and priority assigned to the order in manufacturing facilities. Selection of transportation methods and priority affects cost and duration. An order that is expedited through the network costs more because additional resources are allocated in manufacturing facilities and more expensive transportation methods are implemented.

- Simulation Model is a 'mathematical' replica of the logistics network. This model may contain a set of subsimulation models, each of which represents individual organizational units included in the network, called a distributed simulation model (Fujii, Tsunoda, Ogia, \& Kidani, 1994). The model uses the up-to-date status of the network and runs using predefined scenarios. Each sub-simulation model can be maintained in a local site or all of them can be maintained in a central place. However, the distribution of sub-simulation models should be transparent to the logistics analyst. He/she should be able to define and modify simulation models stored in remote sites through communications software. The final results produced from the model will be passed to the report generator. The logistics analyst can define and change values of variables included in the simulation model through the user interface.

- Report Generator is responsible for providing the logistics analyst with the simulation results in an appropriate format. The logistics analyst can select a display or print format. The logistics analyst should also be able to edit the simulation results through the user interface. The report generator is also responsible for generating the instructions for the customer service department, manufacturing centers, transportation services, and suppliers.

- Network Monitor Agent is intelligent agent software that monitors whether the status of the network has been updated. It performs the following major functions: (1) the agent at a central location looks for changes made in operational information stored at each remote site and obtains the information; (2) the agent at the central location sends the instructions produced by the report generator to the appropriate locations and updates its centrally maintained database; (3) the agent at a remote location notifies the agent at the central location of changes in the status of operations at that site; and (4) the agent at a remote site notifies facility operators at the remote site of instructions from the central location and updates its locally maintained database. This component is critical to make the most recent status of the network available for the simulation model.

- Database Management Software is responsible for maintaining up-to-date status of the network. This is an interface to all information systems used in daily operations. Those information systems provide the database with up-to-date status of operations. Each site can maintain its own database or the database can be centralized. Regardless, the distribution of the database should be transparent to the logistics analyst. In the case that a distributed database is used, appropriate communication software must be utilized to exchange the data between databases. In the case that a centralized database is used, operational data still need to be sent through communication software.

- Databases contain the operational data of the network, simulation results and instructions. Databases at remote sites maintain their operational data and instructions. A database at the central place maintains order information, the simulation results, instructions, as well as some of the local operational data. The operational data includes current status of the manufacturing-logistics network; i.e. the level of raw material inventories, orders being processed and waiting, planned machine repairs, breakdown performance, carrier schedules, and other constraining elements of the network. As in Rogers and Gordon (1993), information is both 'static' (e.g. products, processes, and resource capabilities) and 'dynamic' (e.g. shop floor status, inventories, carrier schedules, carrier space). Status information is required from all the manufacturing centers required by the product ordered and for all the possible combination of transportation services between manufacturing centers.

- Communication Software is responsible for sending and receiving electronic data through telecommunication media.

Figure 3 shows distributed Real-Time Simulation-Based Manufacturing-Logistics Management Systems. In this case, a sub simulation model that represents each of the organizational units, scenarios particular to the site, and a database that contains its operational data, are maintained in the remote site. The database at each site maintains the up-to-date information provided by information systems used in daily operations. The logistics analyst at the central location can use scenario managers and simulation models at remote sites through network to define and modify scenarios and sub-simulation models particular to their sites. 


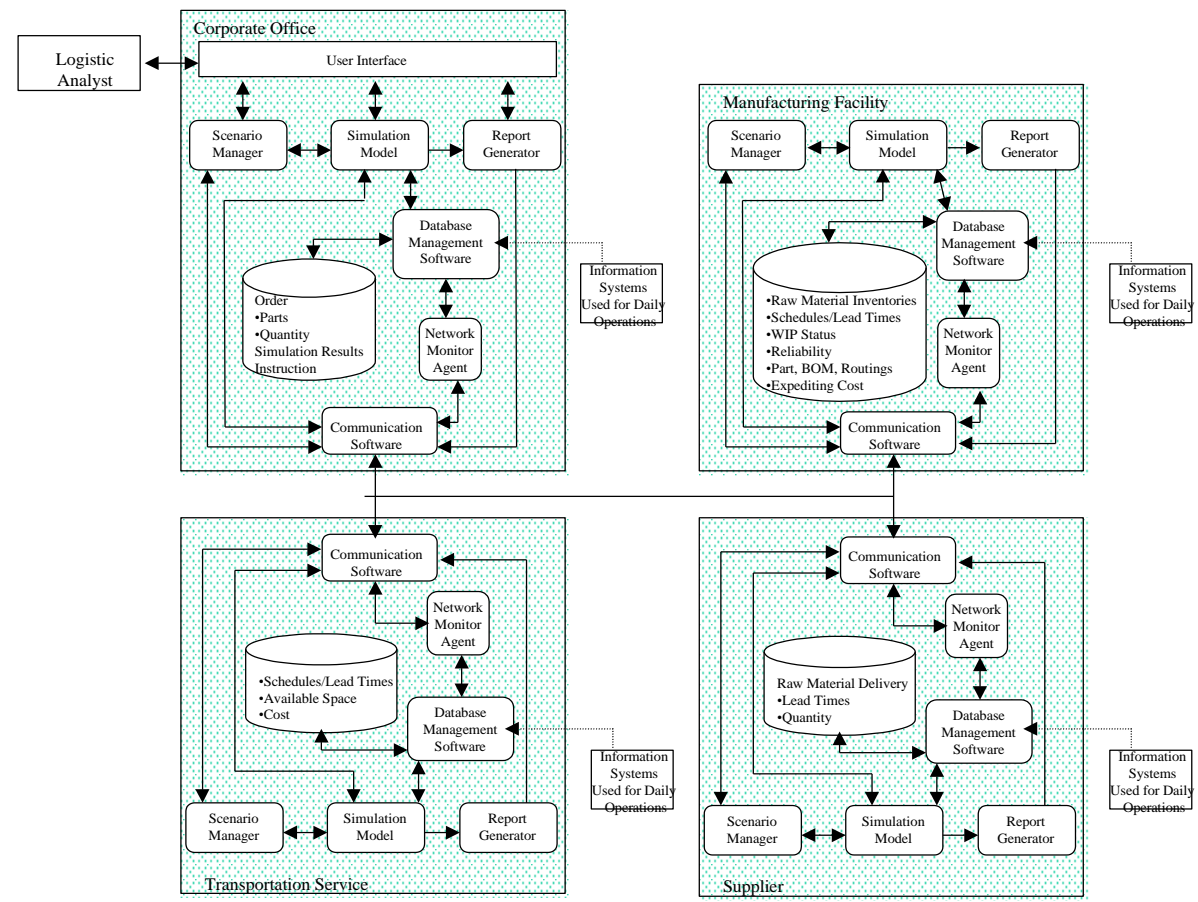

Figure 3: Information Flow In Manufacturing-Logistics Network with RTSMLMS

\subsection{Simulation Model Implementation Issues}

Before a prototype has been developed based on the proposed architecture, issues in implementation of simulation model must have been discussed. Rogers and Gordon (1993) and Harmonosky (1990) point out critical elements required for a successful application of real-time simulation for schedule and control manufacturing systems. Most of these elements apply when the real-time simulation is used in manufacturing-logistics networks. Some elements of particular relevance to this research are:

- Triggering Action: The simulation will be triggered (1) when a new order arrives, (2) when changes in the status of the network are detected, and (3) when the logistics analyst performs a periodical check.

- $\quad$ Alternative Scenarios: These are pre-determined set of scheduling options, product routes, and established transportation modes.

- Update of the Simulation Model Data: Depending on the environment, orders may be limited to a few per day. Also, other changes in the network operations may be limited to a few per week or per month. In those cases, updating can be done only when changes are recognized. On the other hand, if customer orders and other changes in the network are very frequent, the data could be continuously updated (Harmonosky, 1990).

- Length of the Simulation Run: The simulation should be run until all orders currently in the network are completed, including a new order being considered for delivery date assignment. Through this, the logistics analyst can determine the new expected completion time of orders and establishes if the new order or other changes in the network operation data will make any of the current orders late.

- Selection of the Cost/Delivery Date Alternative: Customers of the final product can select the date and cost for them.

- $\quad$ Size of Slack Time ( $k$ ) in Delivery Date Assignment Equation: Some slack time needs to be included for two reasons. First, slack serves as a 'protective' buffer for unexpected disruptions and variation. Second, without some slack, the system has no flexibility that allows orders to be expedited through the network. If all delivery dates are very tight, then adding a 'high priority'/'fast carriers' order may make one or more of the orders already in the network late. Also, it is impossible to expedite the existing orders in the case of emergency caused by unforeseen changes in the network. Thus, the delivery date for an order is described 
by: $\mathrm{d}_{\mathrm{i}}=\mathrm{r}_{\mathrm{i}}+\mathrm{E}\left(\mathrm{C}_{\mathrm{i}}\right)+k$, where $\mathrm{d}_{\mathrm{i}}, \mathrm{r}_{\mathrm{I}}$, and $\mathrm{E}\left(\mathrm{C}_{\mathrm{i}}\right)$ denote the due date, the arrival time, and the expected time required for completing that order (job), respectively.

- The Number of Replications to be Run Under Each Scenario: The determination of $\mathrm{E}\left(\mathrm{C}_{\mathrm{i}}\right)$ depends on how many replications to run. Several replications are necessary in order to take the variability of the operations into account. This helps to obtain a better estimate of the expected completion time. We believe that a fixed number of replications should be pre-set, which provides a reasonable trade-off between time to estimate $\mathrm{E}\left(\mathrm{C}_{\mathrm{i}}\right)$ and the confidence interval of this value. Further information on analyzing simulation results is available in Law and Kelton (1991).

\section{DEVELOPMENT OF A PROTOTYPE}

As the first step to validate our architecture, scenario manager, simulation model, report generator and userinterface were implemented in a prototype. The reason for selecting those components was that other components included in the architecture have been already tested and proven as viable in other research; i.e., 1) distributed database technology and communications software have already been used in the industry for a long time and 2) many types of intelligent agents have been implemented in currently available software.

The prototype was developed for and used in a local manufacturer of made-to-order safety windows with three manufacturing plants and one office location, all of them located within a 50-mile radius. The company has more than 100 employees and 3 million in annual sales. The company holds several patents on impact (bullet proof) and fire resistant glass of unique characteristics. The company produces several types of safety glass. Each product requires relatively similar processes, but there are significant variations in the number of layers added to make a window and types of raw materials used. The process is highly labor-intensive. Due to the manual processes, production times fairly fluctuate. In addition, the yield from the processes also varies and depends on several factors, including raw materials, temperature and humidity, and the ability and experience of operators.

A basic process of the production is presented in Figure 4. When an order has arrived, a large piece of glass is cut to the specified size, then cleaned. Next, each cut piece of glass is prepared to hold a layer of resin or glue. Then film or glass is attached to the glass. The next stage is curing, where the glass is left standing by itself for approximately eight hours. After curing, the glass goes back to the preparation stage for another layer of glue and film or glass. This process is repeated several times according to the order specifications. During these processes, the glass is continuously inspected for lint, particles, and bubbles. At the final stage of manufacturing, the glass is inspected, cleaned and packed. The packaged glasses are shipped to customers scattered throughout the United States through several carriers using several modes of transportation.

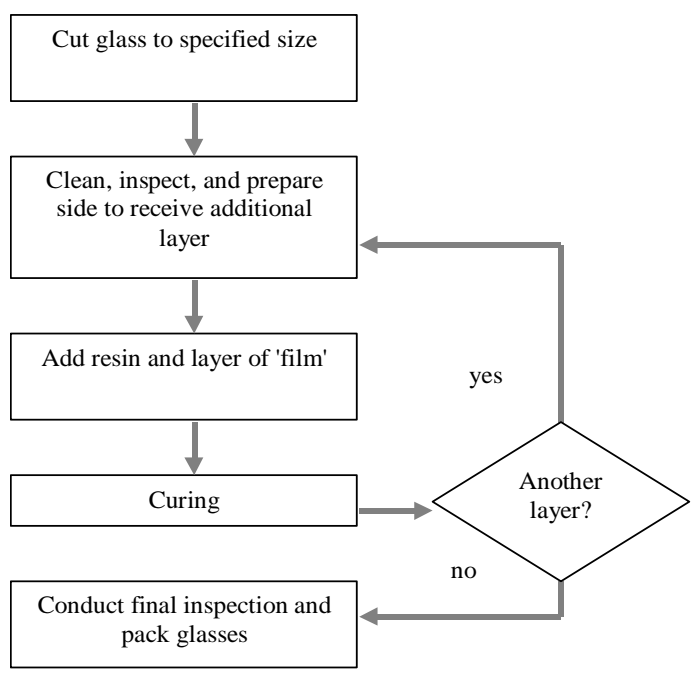

Figure 4: Flow of the Simulated Production Process 
Without the prototype, delivery dates for customer orders (quantities that ranged from less than ten to thousands of windows) were set based on a qualitative match between customer requirements and the perceived load on the network by the sales personnel. This method was effective in most cases when the company was small. The effectiveness of this "perception" method was attributed to the relatively small size of the operation and the high lead times given to customers. However, the increase of demand and associated complexity of the operation were making it harder to determine delivery dates and efficiently manage facilities.

The prototype for this company was developed with Boreland's Delphi 3 application development tool. The simulation model included the manufacturing and logistics factors. The manufacturing factors included in the model are processing lead times and variation, yield, set-up times, and capacity (labor, machines, storage). The logistics factors included in the model are the inventory level and scheduled delivery for the four primary raw materials. Although the operations of suppliers and transportation services were not represented as simulation submodels, the model was a fairly valid representation of the network. The reason that a distributed simulation model was not used was that we focused on validating the most critical components of our architecture using a simple prototype as our initial validation effort. Once we could validate this simple model, we could expand the model into a distributed simulation model.

Information about new customer orders was entered into the prototype by a member of the sales group as they occurred. The operational input data were updated once per day. The prototype must read files sent by e-mails because the facilities were not networked. Although this was not an ideal configuration to test validity of our architecture, we believed this would not affect this initial validation effort significantly.

Three scenarios were specified in the scenario manager: 1) placing an order at the end of the high priority order list; 2) at the end of the normal priority order list, and 3) at the end of the low priority order list. The prototype was designed to run ten replications of each scenario and produce three cost/delivery date options. Management decided on a slack time that depended on the order's priority $(1$ week $=$ high, 3 weeks $=$ normal, 6 weeks $=$ low $)$. If under any of the scenarios the new order made one of the existing orders late, the logistics analyst was notified immediately.

The prototype was also used to monitor and control the current operation. Every time a new order was added, or upon a customer's request for a status report, the prototype simulated the operations of the network and whether the orders currently waiting and being processed could still meet their delivery dates.

The initial assessment of the prototype by management was very positive. The prototype continues to be enhanced for the company. More formal assessment of the prototype will be planned using the enhanced prototype.

\section{SUMMARY}

This paper presented a general architecture for a system that integrates IT and simulation for delivery date assignment and manufacturing-logistics network operations management. The paper also demonstrated the potential of such a system by developing a prototype based on the architecture and implementing it in a small manufacturing company. The following possible advantages of our architecture were identified:

- The architecture can use the most up-to-date operational data to make decisions regarding delivery date assignment and network management.

- $\quad$ The architecture can support both central and distributed environments.

- The prototype developed, based on the architecture, could assign tight delivery dates.

- The prototype could be used to maintain or increase the level of on-time deliveries by monitoring the operations.

- The prototype could generate options on delivery dates and cost based on routings/priority and transportation service options.

We believe that our architecture helps to develop an integrated information system for manufacturinglogistics network management. The architecture incorporates real-time simulation to assign reliable delivery dates and also monitors the operations. It also helps to determine what corrective actions should be taken through the 
simulation. The validation of the architecture was done in a limited environment. The architecture must be validated by developing a full version of a prototype and tested in several companies. Another area we are currently exploring is the incorporation of an optimization agent to the architecture. An optimization agent, as a component of the architecture, is supposed to determine optimized resource allocation for the manufacturing-logistics network.

\section{AUTHOR INFORMATION}

Brian D. Fitzpatrick is a professor of finance for the Helzberg School of Management at Rockhurst University in Kansas City, Missouri. He earned his Ph.D. in Finance with a concentration in Investments from Saint Louis University. Dr. Fitzpatrick spent ten years in the Investment field including positions with two major Wall Street wire houses, as well as serving as senior vice president for both Financial Corporation of America and Commerce Bancshares. Brian has won the Rockhurst University's Teaching Excellence Award, and he has published in nine different fields.

Shahid I. Ali is an Associate Professor of Operations Management for the Helzberg School of Management at Rockhurst University in Kansas City, Missouri. He received his Ph.D. in operations management from Texas Tech University. Dr. Ali's primary research interests are in the areas of petroleum pipeline operations, information systems, and collaborative technology. He is a member of various professional societies and has published in Southern Business and Economic Journal, International Journal of Operations and Quantitative Management, Journal of International Technology and Information Management, Journal of Diversity Management and proceedings of Decision Sciences Institute.

\section{REFERENCES}

1. $\quad$ Ballou, R. H. 1992. Business Logistics Management. New Jersey: Prentice Hall

2. Chang, F. R. 1996. A study of due date assignment rules with constrained tightness in a dynamic job shop. Computers and Industrial Engineering 31-1/2: 205-208.

3. Fujii, S., Tsunoda, H., Ogita, A., and Kidani, Y. 1994. Distributed Simulation Model for Computer Integrated manufacturing. Proceeding of the 1994 Winter Simulation Conference. 946-953.

4. Fry, T. D., P. R. Philipoom, and R. E. Markland. 1989. Due date assignment in a multistage job shop. IIE Transactions 21-2: 153-161.

5. Hameri, A., and A. Paatela. 1995. Multidimensional simulation as a tool for strategic logistics planning. Computers in Industry 27: 273-285.

6. Harmonosky C. M. 1990. Implementation issues using simulation for real-time scheduling, control, and monitoring. Proceeding of the 1990 Winter Simulation Conference, ed. O. Balci, R. P. Sadowski, R. E. Nance: 595-599.

7. Henkoff, R. 1994. Delivering the goods: logistics has become a hot competitive advantage as companies struggle to get the right materials and products to the right place at the right time. Fortune, November 28: 64-78.

8. Law, A. M., and W. D. Kelton. 1991. Simulation Modeling and Analysis. New York: McGraw Hill

9. Lewis, I. and A. Talalayevsky. 1997. Logistics and Information Technology: A Coordination Perspective. Journal of Business Logistics. 18-1: 141-157

10. McConnell, P. G., and D. J. Medeiros. 1992. Real-Time Simulation For Decision Support In Continuous Flow Manufacturing Systems. Proceeding of the 1992 Winter Simulation Conference, ed. J. J. Swain, D. Goldsman, R. C. Crain, and J. R. Wilson.

11. Murphy, P.R. and J. M. Daley. 1996. International Freight Forwarder Perspectives on Electronic Data Interchange and Information Management Issues. Journal of Business Logistics. 17-1: 63-84.

12. Rogers, P., and R. J. Gordon. 1993. Simulation for real-time decision making in manufacturing systems. Proceeding of the 1993 Winter Simulation Conference, ed. G. W. Evans, M. Mollaghasemi, E. C. Biles.

13. Rohrer, M. 1997. Seeing is believing: The importance of Visualization in Manufacturing Simulation. IIE Solutions 29-5: 24-28. 
NOTES 